\title{
The hypergiant masers: episodic mass loss, convection and magnetic fields
}

\author{
Roberta M. Humphreys \\ Astronomy Department, University of Minnesota, Minneapolis, MN 55455, USA
}

\begin{abstract}
The cool hypergiants are the most luminous known stars in the upper HR Diagram in the apparent temperature range represented by spectral types A to M. Most of the stars in this regime are unstable as evidenced by their high mass loss rates, variability, and in some cases large IR excesses and circumstellar ejecta. We have obtained high resolution multi-wavelength images with HST/WFPC2 of several of the most known evolved cool stars including several well known stellar masers. VX Sgr and S Per were marginally resolved, while NML Cyg has a peculiar asymmetric envelope that has been shaped by its environment. The powerful maser sources IRC+10420 and VY CMa have extensive and complex circumstellar ejecta due to high mass loss episodes apparently driven by large-scale convective activity.
\end{abstract}

Keywords. supergiants, stars: winds, outflows, stars: activity

\section{Introduction}

To better understand the evolution of luminous, cool evolved stars and their mass loss mechanism, my collaborators and I have been obtaining high resolution imaging and spectroscopy of several of the stars called "cool hypergiants". These stars lie on or near the empirical upper luminosity boundary in the HR Diagram (Humphreys \& Davidson 1994, de Jager 1998). They were selected based on their infrared excess emission, high mass loss rates, molecular emission, and photometric and spectroscopic variability.

The "yellow" hypergiants, $\rho$ Cas, HR 5171a and HR 8752 and the M-type supergiant $\mu$ Cep are all point sources, while VX Sgr, S Per, NML Cyg, VY CMa, and IRC+10420 were all resolved and the latter three have circumstellar ejecta. Note that all five of these stars are strong maser sources. VX Sgr and S Per have marginally resolved envelopes comparable is size to their dust formation radii. S Per's circumstellar nebula appears elongated in a NE/SW orientation similar to that for its $\mathrm{OH}$ and $\mathrm{H}_{2} \mathrm{O}$ masers, while VX Sgr is embedded in a spheroidal envelope. NML Cyg, IRC +10420 and VY CMa are discussed in more detail below

\section{The post red supergiant - IRC $+\mathbf{1 0 4 2 0}$}

With its high luminosity of $L \sim 5 \times 10^{5} L_{\odot}$ (Jones et al. 1993), extraordinary mass loss rate of $3-6 \times 10^{-4} M_{\odot} \mathrm{yr}^{-1}$ (Knapp \& Morris 1985, Oudmaijer et al. 1996, Humphreys et al. 1997), and intermediate temperature, IRC+10420 is one of the the most important evolved stars in the upper HRD. It is also one of the warmest maser sources known.

Our HST images of IRC+10420 (Humphreys et al. 1997) revealed a complex circumstellar environment, with a variety of structures including condensations or knots, ray-like features, and semi-circular arcs or loops within 2 " of the star which are evidence for high mass loss ejections in the past few hundred years (Figure 1). One or more distant reflection shells at 5 " to $6 "$ from the star were ejected $\approx 3000$ yrs ago, possibly when it was still a red supergiant. 


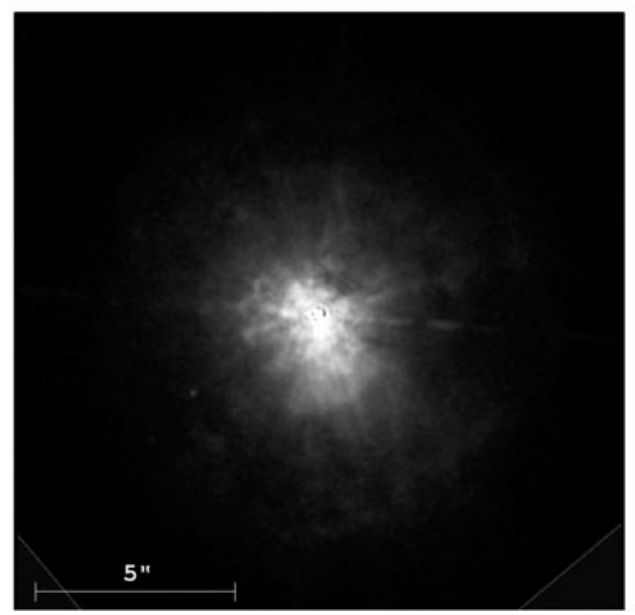

Figure 1. HST/WFPC2 image of IRC +10420 showing its complex inner ejecta.

IRC +10420's OH maser emission is peculiar with a complex and clumpy distribution within 1.5" of the star and demonstrating complex variations in its intensity with time (Lewis et al. 1986). Although it is a strong $\mathrm{OH}$ source, it is not a known source of $\mathrm{H}_{2} \mathrm{O}$ and $\mathrm{SiO}$ emission common in many evolved late-type stars. This may be because it is so warm. IRC +10420 may not be of much interest as a source of abundant maser emission, but it is an extremely important star for understanding the final stages of massive star evolution.

\section{NML Cyg - Interacting with its environment}

The powerful OH/IR source NML Cyg is also near the empirical upper luminosity boundary for red supergiants with a luminosity of $5 \times 10^{5} L_{\odot}$ and a mass loss rate of $6.4 \times 10^{-5} M_{\odot} y r^{-1}$ (Hyland et al. 1972, Morris \& Jura 1983). It is only $\sim 100 \mathrm{pc}$ from the large association Cyg OB2 in the Cygnus X superbubble (Morris \& Jura 1983, Knodlseder 2003).

Our HST/WFPC2 images (Schuster et al. 2006) show that NML Cyg has a small but very obvious circumstellar nebula with a peculiar asymmetric shape (Figure 2). There are remarkable similarities between the asymmetric envelope that we see and the much more distant ( $\sim 30 "$ from the star) H II contours around NML Cyg (Habing et al. 1982). Morris \& Jura (1983) showed that the asymmetric "inverse" H II region was the result of the interaction of a spherically symmetric, expanding wind from NML Cyg and photoionization from Lyman continuum photons from the luminous, hot stars in Cyg OB2. Our images show circumstellar material much closer to NML Cyg than the surrounding $\mathrm{H}$ II region and coincident with the water masers as well as $\mathrm{SiO}$ masers, suggesting that we are likely imaging the photo-dissociation boundaries. Schuster et al. have modeled the molecular photodissociation boundary shown superposed on the image of NML Cyg. Note that the overall distribution of the $\mathrm{H}_{2} \mathrm{O}$ masers is not only similar in extent to the reflection nebula, but also matches its convex shape. Thus, it appears that the molecular material SE of the star is preferentially shielded from photo-dissociation. 


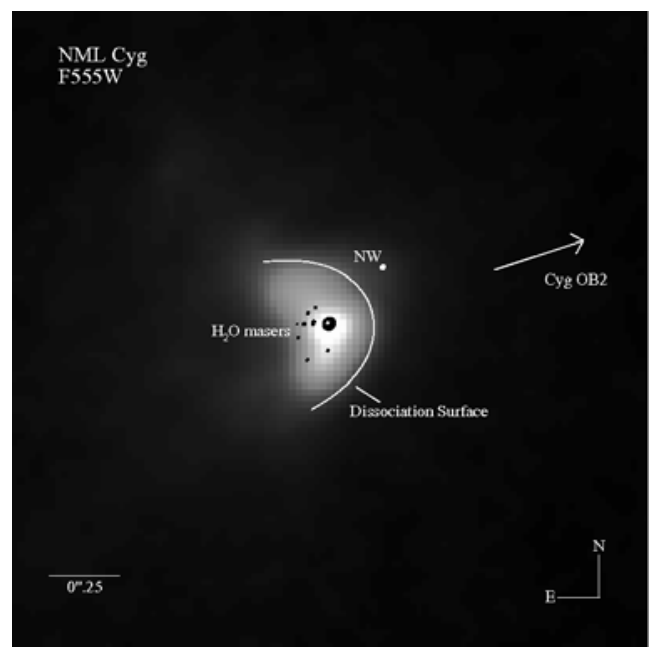

Figure 2. HST/WFPC2 image of NML Cyg with the photodissociation boundary and positions of the $\mathrm{H}_{2} \mathrm{O}$ masers superposed.

\section{The extreme red hypergiant - VY CMa}

The extreme red supergiant and powerful infrared source and $\mathrm{OH}$ maser, VY CMa is one of the most luminous and largest evolved stars known. At its distance of $1.5 \mathrm{kpc}$ (Herbig 1972; Lada \& Reid 1978; Marvel 1997), VY CMa's luminosity is $\approx 4.3 \times 10^{5} L_{\odot}$. Its very visible asymmetric nebula, 10" across, combined with its high mass loss rate of $4 \times 10^{-4} M_{\odot} y r^{-1}$ (Danchi et al. 1994), makes VY CMa a special case even among the cool hypergiants that define the upper luminosity boundary.

High resolution imaging with HST (FOC, Kastner \& Weintraub 1998; WFPC2, Smith et al. 2001) and near-IR interferometry (Monnier et al 1999) have revealed VY CMa's complex circumstellar environment. The multi-wavelength HST/WFPC2 images of VY CMa (Smith et al. 2001) showed the complexity of detail in its ejecta (Figure 3) including the prominent arc to the northwest, two bright filamentary arcs to the southwest, plus relatively bright clumps of dusty knots near the star, and numerous small arcs throughout the nebula. All of which are evidence for multiple and asymmetric mass loss episodes. The apparent random orientations of the arcs suggested that they were produced by localized ejections, possibly from large-scale convective regions on the star.

The extensive maser $\left(\mathrm{OH} . \mathrm{H}_{2} \mathrm{O}\right.$, SiO), and $\mathrm{CO}$ observations, however, have been interpreted as evidence for an axis of symmetry with possible bipolar outflows and a disk-like distribution for the circumstellar material. The $\mathrm{OH}$ maser intensity maxima are within $0.5 "$ and are distributed along a NE-SW axis at a position angle of $\sim 50^{\circ}$ (Bowers et al. 1983). If there is a NE-SW polar/rotation axis, then Arcs 1 and 2 could both be part of an associated bipolar outflow depending on the opening angle of the cone, but from different locations on the star corresponding to their spatial separation.

The $\mathrm{H}_{2} \mathrm{O}$ maser spots appear to be oriented much more east-west (Richards, Yates \& Cohen 1998). The highly polarized SiO maser spots (Shinaga et al. 2004) have a 0.2" north-south distribution on the sky, although the $\mathrm{SiO}$ emission is bipolar with a mean position angle of $72^{\circ}$. Similarly, diffraction - limited speckle interferometry in the near infrared also shows a primarily north-south orientation for the dust shell extended 0.2" with a position angle $\sim 153$ to $176^{\circ}$ (Wittkowski, Langer \& Weigelt 1998), while near-IR aperture-masked interferometry (Monnier et al. 1999) showed a southward extension of the dust emission within 0.1 " but no clear disklike or bipolar geometry in the images. 


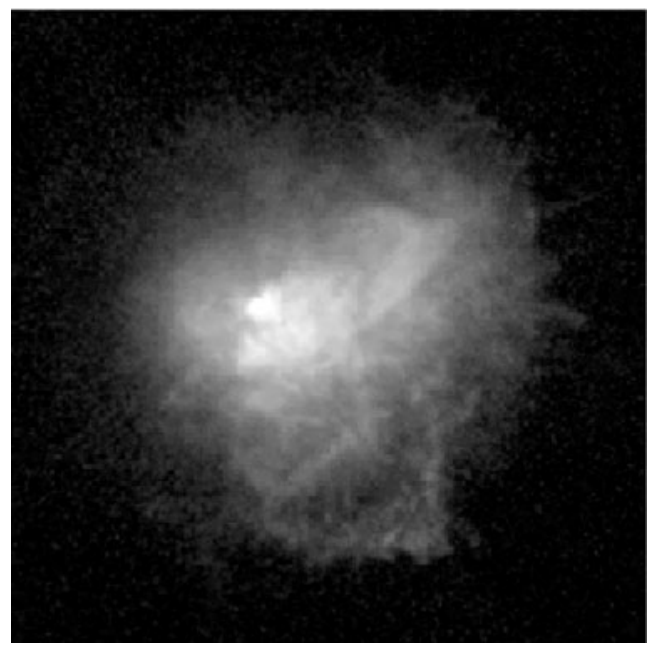

Figure 3. HST/WFPC2 image of showing the prominence-like arcs and clumps of knots in its ejecta.

K-band interferometric measurements however, indicate a bipolar distribution within 0.1 " of the star but with the dusty disk oriented east-west (Monnier et al. 2004). Recent interferometric millimeter observations of CO and SO (Muller et al. 2006) have been modelled as a bipolar outflow in the east-west direction, but with a very wide opening angle $\left(\sim 120^{\circ}\right)$ and an expanding shell elongated north-south.

The maser maps and interferometric observations are thus not always consistent with each other. With this lack of strong evidence for a well-defined axis of symmetry or bipolar axis in VY CMa, we conclude that the morphology and kinematics of the numerous arcs and knots are more consistent with a history of localized mass ejections from active regions on the star not strongly aligned with a presumed axis or equator.

\section{Convective activity and magnetic fields}

We have recently obtained second epoch images and polarimetry of VY CMa with the HST (Humphreys et al. 2007, Jones et al. 2007). The polarization together with the color from the images can yield information on the line of sight distribution of the nebulosity and let us determine the relative locations of the arcs and knots, while the transverse motions combined with the Doppler velocities (Humphreys et al. 2005) provide a complete picture of the kinematics of the ejecta including the total space motions and directions of the outflows. Our results show that the arcs and clumps of knots are moving at different velocities, in different directions, and at different angles relative to the plane of the sky and to the star, confirming their origin from eruptions at different times and from physically separate regions on the star, not by a more uniform long-term mass loss. They represent localized, relatively massive (few $\left.\times 10^{-3} M_{\odot}\right)$ ejections. We are suggesting that they are the result of activity associated with large convective regions or starspots, which have now been observed on numerous red giants, AGB stars and supergiants.

Recently, Vlemmings et al. $(2002,2005)$ have estimated the magnetic field strength from the circular polarization of $\mathrm{H}_{2} \mathrm{O}$ masers in the ejecta of AGB stars and several evolved supergiants including the strong OH/IR sources VY CMa, VX Sgr, NML Cyg, and S Per. They report magnetic fields in VY CMa of $\sim 200 \mathrm{mG}$ at distances of 220 AU. Their analysis supports the Zeeman interpretation of the circular polarization of the 
$\mathrm{SiO}$ masers only a few AU from the surface of the stars (Barvainis et al. 1987; Kemball \& Diamond 1997). Together with Zeeman splitting of the $\mathrm{OH}$ emission far out in the wind at a few thousand AU (Szymczak \& Cohen 1997; Masheder et al. 1999), these measurements confirm the presence of a magnetic field throughout the ejecta of VY CMa. The $\mathrm{OH}$ measurements at distances in the ejecta comparable to the prominencelike arcs, imply a magenetic field at the star of $~ 8000$ G. Similarly, circular polarization of the $\mathrm{OH}$ emission in IRC +10420 (Nedoluha \& Bowers 1992) suggests a field strength of $\sim 3000 \mathrm{G}$ at the star.

These values seem too large for a global magnetic field but may be representative of the field associated with the active regions.

\section{Acknowledgements}

It is a please to thank my collaborators on these studies, Kris Davidson, Robert Gehrz, Andrew Helton, George Herbig, Terry J. Jones, Massimo Marengo, Gerald Ruch, Michael Schuster, Nathan Smith and George Wallerstein.

\section{References}

Barvainis, R., McIntosh, G. \& Predmore, C. R. 1987, Nature 329, 613

Bowers, P. F., Johnston, K. J., \& Spencer, J. H . 1983, ApJ 274, 733

Danchi, W. C., Bester, M., Degiacomi, C. G., Greenhill, L. J., \& Townes, C.H. 1994, AJ 107, 1469

de Jager, C. 1998, AEAR 8, 145

Habing, H. J., Goss, W. M., \& Winnberg, A. 1982, A\&\&A 108, 412

Herbig, G. H. 1972, ApJ 172, 375

Humphreys, R. M. \& Davisdon, K. 1994, PASP 106, 1025

Humphreys, R. M., Smith, N., Davidson, K., Jones, T. J. et al. 1997, AJ 114, 2778

Humphreys, R. M., Davidson, K., Ruch, G., \& Wallerstein, G. 2005, AJ 129, 492

Humphreys, R. M., Helton, L. A., \& Jones, T. J. 2007, $A J$ in press

Jones, T. J., Humphreys, R. M., Gehrz, R. D. et al. 1993, ApJ 411, 323

Jones, T. J., Humphreys, R. M., Helton, L. A., Gui, C. \& Xiang, H. 2007, AJ in press

Kastner, J. H. \& Weintraub, D. A. 1998, AJ 115, 1592

Kemball, A. J. \& Diamond, P. J. 1997, ApJ Letters 481, L111

Knapp, G. R. \& Morris, M. 1985, ApJ 292, 640

Knödlseder, J. 2003, IAU Symp. 212, A Massive Star Odyssey, from Main Sequence to Supernova, eds. K.A. van der Hucht, A. Herrero \& C. Esteban, 505

Lada, C. J. \& Reid, M. J. 1978, ApJ 219, 95

Marvel, K.B. 1997, PASP 109, 1286

Masheder, M. R. W. et al. 1999, New Astron 43, 563

Monnier, J. D., Tuthill, P. G., Lopez, B., Cruzalebes, P. et al. 1999, ApJ 512, 351

Monnier, J. D. et al. 2004, APJ 605, 436

Morris, M. \& Jura, M. 1983, APJ 267, 179

Muller, S., Dinh-V-Trung, Lim, J. Hirano, N., Muthu, C, \& Kwok , S. 2007, ApJ 656, 1109

Nedoluha, G. E. \& Bowers, P. F. 1992, ApJ 392, 249

Oudmaijer, R. D., Groenewegen, M. A. T., Matthews, H. E., et al. 1996, MNRAS 280, 1062

Richards, A. M. S., Yates, J. A., \& Cohen, R. J. 1998, MNRAS, 299, 319

Shinnaga, H., Moran, J. M., Young, K. H. \& Ho, P. T. P. 2004, ApJ Letters 616, L47

Schuster, M. T., Humphreys, R. M., \& Marengo, M 2006, AJ 131, 603

Smith, N., Humphreys, R. M., Davidson, K., et al. 2001, AJ 121, 1111

Szymczak, M. \& Cohen, R. J. 1997, MNRAS 288, 945

Vlemmings, W. H. T., Diamond, P. J. \& van Langevelde, H. J. 2002, A\&SA 394, 589

Vlemmings, W. H. T., van Langevelde, H. J., \& Diamond, P. J. 2005, Mem. Soc. Astr. It. 76, 462

Wittkowski, M., Langer, N., \& Weigelt, G. 1998, A\&A 340, L39 